\title{
O.S.P.
}

L'orientation scolaire et professionnelle

$32 / 1 \mid 2003$

Varia

\section{Taylor. Les Sources du moi-La formation de l'identité moderne}

Paris : Le Seuil

Barbara Ritz

\section{OpenEdition}

Journals

Édition électronique

URL : http://journals.openedition.org/osp/3223

DOI : $10.4000 /$ osp.3223

ISSN : 2104-3795

Éditeur

Institut national d'étude du travail et d'orientation professionnelle (INETOP)

Édition imprimée

Date de publication : 1 mars 2003

Pagination : 167-169

ISSN : 0249-6739

Référence électronique

Barbara Ritz, «C. Taylor. Les Sources du moi-La formation de l'identité moderne », L'orientation scolaire et professionnelle [En ligne], 32/1 | 2003, mis en ligne le 06 mai 2011, consulté le 21 septembre 2020.

URL : http://journals.openedition.org/osp/3223 ; DOI : https://doi.org/10.4000/osp.3223

Ce document a été généré automatiquement le 21 septembre 2020.

(c) Tous droits réservés 


\title{
C. Taylor. Les Sources du moi-La formation de l'identité moderne
}

\author{
Paris : Le Seuil
}

Barbara Ritz

\section{RÉFÉRENCE}

Paris : Le Seuil

1 Charles Taylor est un philosophe canadien qui s'intéresse au concept de l'identité et aux problèmes que posent les questions d'intégration ethnique et culturelle de populations diverses. Il est connu pour être un penseur majeur du multiculturalisme et du communautarisme. Ses positions sont engagées dans des combats qui touchent aux grands phénomènes de société. Taylor théorise et philosophe dans ce sens. Les questions autour de la morale sont à ses yeux, des incontournables et, selon lui, il existerait une diversité de conception de la «vie bonne» dans laquelle il faudrait rendre justice à deux notions : la différence et l'unité. Ses ouvrages principaux sont : le Malaise de la modernité (1994), la Liberté des modernes (1997), les Sources du moi (1998).

Dans les Sources du moi, Charles Taylor se donne pour objectif de définir l'identité moderne et d'en écrire l'histoire. Derrière ce travail, la finalité est toujours pour Taylor de nourrir sa compréhension des problématiques modernes et des nouveaux phénomènes de société. Pour cela, l'auteur choisit de retracer la genèse de notre identité «de» moderne en insistant sur le fait qu'elle nous vient de l'héritage des époques passées et de la longue histoire des idées qui nous a été léguée. Pour Taylor, cette histoire est complexe et riche et elle peut être source de tensions. Elle se lit, se déchiffre et s'analyse à partir des non-dits et des présupposés des courants actuels de la pensée moderne et post-moderne. C'est ce travail d'explicitation et de réflexion qu'il s'est proposé de poursuivre. Sa démarche reste essentiellement analytique et historique. Son entreprise est d'autant plus considérable qu'elle a nécessité un travail d'érudition hors du commun. À ce titre, cette œuvre peut être qualifiée de magistrale. 
3 Selon Taylor, notre identité moderne reposerait sur des idéaux et des interdits qui modèlent notre pensée, notre épistémologie, ainsi que notre philosophie de moderne, sans que nous en ayons conscience. Ces idéaux, développés par les grands courants philosophiques, continuent bien plus que nous ne l'imaginons de façonner notre identité. Selon cet auteur, nous serions les héritiers d'innombrables représentations, très souvent informulées, qui conditionnent fortement notre conception moderne du fait d'être un "agent humain ». Derrière cette expression, Taylor veut souligner les différentes dimensions qui s'y rattachent : le sens de l'intériorité, le sens de la liberté, le sens de l'individualité et le sentiment d'appartenir à la nature.

4 C'est à partir de cette mise en perspective générale de notre identité que Taylor veut réinterroger la notion de modernité afin de mieux en cerner les principaux enjeux. Des auteurs comme Foucault, Habermas, Mac Intyre et d'autres ont tenté d'apporter leurs réponses mais Taylor les juge trop insatisfaisantes. Dans ce livre, il prend nettement position, sa pensée se veut forte. Son objectif est, clairement, de réfuter des points de vue trop optimistes, ou au contraire trop insistants sur la décadence de nos sociétés. Taylor cherche, au contraire, à dépasser ces oppositions qu'il juge stériles, pour saisir l'essence même de la présence simultanée d'un sentiment de grandeur et de misère, voire de danger, qui définit la spécificité de notre époque moderne.

5 Par ailleurs, Taylor prône l'idée d'une morale réaliste intégrant les éléments de l'histoire et de la psychologie. Son but est de lutter contre les utopies les plus désastreuses, sans toutefois renoncer aux exigences de la morale. Il cherche à redonner un sens positif et renouvelé au terme de "morale», pour qu'elle puisse s'incarner réellement dans les actes du quotidien. Sa réflexion se fonde sur une anthropologie philosophique qui proposerait une nouvelle ontologie de la morale, en réaction à la prééminence actuelle de l'épistémologie et des préceptes de la sociobiologie et du relativisme pragmatique issu du naturalisme, sur lesquels cette épistémologie se fonde.

Pour Taylor, trois aspects principaux fonderaient l'identité moderne, c'est-à-dire :

1. Le sentiment d'intériorité. Celui-ci renvoie à la dimension de profondeur de l'individu. Cette conception autour de l'homme a une histoire, que Taylor revisite de Saint-Augustin à Descartes, puis de Montaigne à nos jours. À la suite du «désenchantement progressif de notre monde, de la perte du Logos et de la disparition d'un ordre extérieur et immuable qui s'imposerait à nous ", notre idéal moral s'est enraciné au fil de l'histoire et des évolutions culturelles, dans la quête et l'affirmation d'une plus grande authenticité (histoire, traditions et culture), ainsi que dans le déplacement toujours plus important vers l'intérieur de l'individu, afin de trouver ce qui fonde et crée notre sentiment moderne d'identité personnelle, "où chacun est devenu sa propre mesure ». Toutefois, si le monde n'est plus porteur du Logos et si le sens des choses se construit à partir de l'intériorité de l'individu, les relations inter et intra personnelles, sont prises dans des réseaux spécifiques de communication et de signifiance, liées aux caractéristiques de l'environnement. C'est en cela que Taylor précise que les sources du moi ne sont pas dans le moi lui-même, car il ne peut exister d'identité en dehors de cadres extérieurs à soi.

2. L'affirmation de la vie quotidienne ordinaire est une expression technique qui désigne la vie de production et la vie de famille. Auparavant, la vie de production était seulement une condition nécessaire de vie et la vie n'avait de sens qu'en tant que « vie bonne». Depuis la Réforme des Lumières, les formes «supérieures » de vie, comme la noblesse, la guerre, le civisme militant, la contemplation, l'ascétisme héroïque, trop souvent réservées aux élites, sont rejetées au profit de l'affirmation des valeurs de production, de travail et de construction d'une famille, présentes dans la vie quotidienne de chacun. Les valeurs « supérieures » de la vie ne se situent plus maintenant en dehors de la vie quotidienne mais 
dans une manière de la vivre. Cette transformation radicale dans le choix des valeurs s'est accompagnée d'un sens aigu accordé à la souffrance et au bien-être de l'individu, à l'importance de l'autonomie et à la signification que l'on a développé du respect de la vie de tous et de l'intégrité humaine, dans l'Occident moderne.

3. La voix de la nature. Après le radicalisme rationnaliste des Lumières, le $18^{\mathrm{e}}$ siècle renoua avec une certaine orientation face à la nature qui n'est pas sans rapport avec une position déiste et un nouveau sentiment de confiance en l'ordre naturel. La conception apparaît «d'un nouveau sentiment quasi panthéiste de l'existence d'un esprit cosmique qui parcourerait l'ensemble de la nature et s'exprimerait dans l'humanité ». L'expression des sentiments, de la subjectivité, l'imagination, la créativité, et l'émerveillement face à la Beauté de la nature sont exaltés. Les courants expressivistes et romantiques de cette époque imprègneront fortement les esprits, particulièrement en Allemagne. Les profondeurs et la bonté de la nature et de l'ordre des choses vont aussi se manifester à l'intérieur de ce qui procède de notre nature propre, de nos désirs et de nos sentiments intimes. Ce mouvement participe de l'idée d'une morale autonome dont les sources se situent désormais en nous.

7 S'agissant de l'idée du Bien et de la Morale, Taylor refuse la différence entre faits et valeurs. Il considère que notre rapport au monde consiste à interpréter sans cesse notre orientation vers le Bien. Pour lui, à l'inverse de Rawls, le Bien prime sur le juste. L'auteur propose ses propres définitions de ce qu'il appelle «Bien(s)». Elles sont incontournables pour comprendre sa pensée. Par «Biens», nous pouvons entendre ce qui peut donner un sens fort à sa vie et proposer ainsi des normes qui orientent les désirs et les aspirations de chacun. Pour Taylor, avoir une identité, c'est pouvoir donner un sens minimal à sa vie à travers une mise en récit de soi et pouvoir juger, selon des "discriminations qualitatives" ou des "évaluations fortes", ce que l'on considère comme supérieur, ou comme ayant de la valeur pour soi. Taylor parle aussi de biens de vie, de biens convergents, de biens communs, de biens constitutifs ou encore d'hyperbiens. Les biens constitutifs et les hyperbiens sont des biens d'ordre supérieur, qui peuvent entrer en conflit les uns avec les autres en fonction de leur nature. Le pluriel prémunit ici du déploiement du fanatisme.

En conclusion, pour Taylor, le défi fondamental de la modernité consiste à réussir à concilier deux types de revendications humaines : l'appartenance à une communauté humaine d'un côté et, de l'autre, la reconnaissance d'une diversité d'individus singuliers, ainsi que de l'irréductible dignité de la personne. 